\title{
RHEUMATOID ARTHRITIS ASSOCIATED WITH OSLER-WEBER-RENDU DISEASE: FIRST DESCRIPTION
}

Caio Marcos Ribeiro Gonçalves ${ }^{1}$, Carlos Ewerton Maia Rodrigues ${ }^{2,3}$, Cristiano Augusto de Freitas Zerbini4, Jozélio Freire de Carvalho ${ }^{5, *}$

1.Universidade Salvador, Salvador (BA), Brazil. 2.Universidade de Fortaleza, Fortaleza (CE), Brazil. 3.Universidade Federal do Ceará, Fortaleza (CE), Brazil. 4.Centro Paulista de Investigação Clínica, São Paulo (SP), Brazil. 5.Universidade Federal da Bahia, Salvador (BA), Brazil.

*Corresponding author: jotafc@gmail.com

\section{BACKGROUND}

Rheumatoid arthritis (RA) is a common systemic inflammatory disease, which mainly affects the peripheral joints. Osler-WeberRendu disease (OWR), also known as hereditary hemorrhagic telangiectasia (HHT), is a rare autosomal dominant genetic disorder that leads to abnormal blood vessel formation in the skin, mucous membranes, and often in organs such as the lungs, liver, and brain. Until now there is no reported case describing the concomitance of both pathologies. The objective was to describe a clinical case of RA associated with OWR.

\section{CASE REPORT}

A 63-year-old female patient had several recurrent episodes of intestinal hemorrhage and started episodes of epistaxis, with normal platelets. She underwent colonoscopy that revealed vascular ectasias, and received blood transfusions. Hemostasis was performed with argon laser (2 sessions). The computed tomography angiography revealed an enlarged liver with arteriovenous fistulas visualized throughout the parenchyma and some areas with small nodules and also arteriovenous fistula in the upper lingual region of the lung. Dermatoscopy revealed telangiectasias on her fingers and on her tongue and lips. She had a family history of epistaxis in his father and two brothers, one of them with hemoptysis and diagnosis of pulmonary arteriovenous fistula. The TTH genetic test was positive for OWR. The patient fulfilled the diagnosis criteria for OWR. She evolved well, without further episodes of intestinal hemorrhage, maintaining mild episodes of epistaxis. In 2013, she was diagnosed with rheumatoid arthritis, she had polyarthritis and positive anti-CCP, with increased levels of CRP. She was treated with rituximab and achieved a complete clinical remission.

\section{CONCLUSION}

The patient with RA in use of rituximab who had a previous history of OWR is herein described and this is the first case report of a patient diagnosed with RA who had OWR disease. 